\title{
Systemic lupus erythematosus in Zimbabwe
}

\author{
H G TAYLOR AND C M STEIN ${ }^{2}$ \\ From the ${ }^{1}$ Mpilo Hospital, Bulawayo, Zimbabwe; and the ${ }^{2}$ Department of Clinical Pharmacology, University \\ of Zimbabwe, Harare, Zimbabwe
}

SUMmaRY Systemic lupus erythematosus (SLE) was diagnosed in 31 black Zimbabweans over a six year period. Renal involvement (71\%) was more common and photosensitivity (16\%) and serositis $(23 \%)$ less common than in the United States. Lymphopenia (48\%) was the commonest haematological abnormality. Unusual complications included subarachnoid haemorrhage, cardiac rhythm disturbance, portal and superior mesenteric vein thrombosis, and a non-Hodgkin lymphoma. Tuberculosis was a common differential diagnosis that was difficult to exclude. Nine patients $(29 \%)$ died within one year of diagnosis. SLE is being recognised more commonly in Zimbabwe.

Key word: Africa.

Systemic lupus erythematosus (SLE) is rare in black Africans and was not recorded before $1960 .{ }^{1}$ After sporadic reports from Uganda ${ }^{23}$ and Nigeria ${ }^{4}$ Jessop and Meyers found eight cases in 11 years ${ }^{5}$ and Seedat and Pudifin 17 cases in six years in South Africa. ${ }^{6}$ In 1980 Kanyerezi et al reported on 21 patients with SLE seen in 11 years at Mulago Hospital, Uganda. ${ }^{7}$

A five year prospective study of polyarthritis in Zimbabwe reported in 1969 showed no cases of SLE. ${ }^{8}$ Since little is known about SLE in Africa and the clinical features of the disease in Zimbabwe have not been recorded we report our findings.

\section{Patients and methods}

The records of all black patients with SLE diagnosed for the first time at Mpilo Hospital in Bulawayo and Harare and Parirenyatwa Hospitals in Harare from January 1979 to December 1983 were reviewed. In addition, patients diagnosed as having SLE at the same hospitals in 1984 were seen prospectively. The American Rheumatism Association (ARA) revised criteria ${ }^{9}$ were used for inclusion in the study.

\section{Results}

The ARA criteria were fulfilled by 31 patients, 30

Accepted for publication 14 February 1986.

Correspondence to Dr H G Taylor, PO Box MR 109, Marlborough, Harare, Zimbabwe. female and one male, whose average age at diagnosis was 28 years (range 13-46 years). Of these 31 patients, 22 stated that they lived in either Bulawayo or Harare and the remaining nine were referred from outlying districts.

CLINICAL FEATURES

Table 1 lists the ARA criteria and the relative frequency of each in our patients and in the multicentre American study on which the revision of the criteria was based. ${ }^{9}$

S K I N

In addition to the rashes listed as criteria, nonspecific rashes were seen in six patients (two macular, two maculopapular, and two urticarial), and one patient had longstanding psoriasis. Alopecia and Raynaud's phenomenon occurred in 10 and five patients respectively. Skin biopsies were performed in two patients and showed changes compatible with SLE.

VASCULAR FEATURES

Vasculitic lesions of the peripheries were seen in five patients, one of whom suffered a subarachnoid haemorrhage presumably due to vasculitis. Deep vein thrombosis of the leg occurred in one patient, and another patient was found to have thrombosis of the portal and superior mesenteric veins at autopsy. A biopsy specimen showed organising thrombus with a marked inflammatory cell infiltrate in the vessel walls. 
Table 1 Frequency of 1982 revised ARA criteria in Zimbabwean blacks with SLE compared with a multicentre American study

\begin{tabular}{|c|c|c|c|c|}
\hline \multirow[t]{2}{*}{ Criteria } & \multicolumn{2}{|c|}{$\begin{array}{l}\text { Present series } \\
(n=31)\end{array}$} & \multicolumn{2}{|c|}{$\begin{array}{l}\text { Tan et al } \\
(n=177)\end{array}$} \\
\hline & No & $\%$ & No & $\%$ \\
\hline Malar rash & 19 & 61 & 101 & 57 \\
\hline Discoid rash & 6 & 19 & 31 & 18 \\
\hline Photosensitivity & 5 & $16^{* *}$ & 76 & 43 \\
\hline Oral ulcers & 6 & 19 & 47 & 27 \\
\hline Arthritis & 25 & 81 & 152 & 86 \\
\hline Serositis & 7 & $23^{* *}$ & 99 & 56 \\
\hline Pleuritis & 5 & 16 & 92 & 52 \\
\hline Pericarditis & 3 & 10 & 31 & 18 \\
\hline Renal disorder & 22 & $71^{*}$ & 91 & 51 \\
\hline Proteinuria & 16 & 52 & 89 & 50 \\
\hline Cellular casts & 16 & 52 & 64 & 36 \\
\hline Neurological disorders & 4 & 13 & 35 & 20 \\
\hline Seizures & 2 & 6 & 21 & 12 \\
\hline Psychosis & 2 & 6 & 22 & 12 \\
\hline Haematological disorder & 19 & 61 & 105 & 59 \\
\hline Haemolytic anaemia & 4 & 13 & 31 & 18 \\
\hline Leucopenia & 6 & 19 & 82 & 46 \\
\hline Lymphopenia & 15 & 48 & - & - \\
\hline Thrombocytopenia & 3 & 10 & 37 & 21 \\
\hline Immunological disorder & 20 & 65 & 149 & 84 \\
\hline $\begin{array}{l}\text { Positive LE cell } \\
\text { preparation }\end{array}$ & $16 / 26^{\dagger}$ & 62 & $58 / 79^{+}$ & 73 \\
\hline Anti-DNA & $7 / 7^{+}$ & 100 & $113 / 168^{\dagger}$ & 67 \\
\hline Anti-Sm & - & - & $34 / 108^{\dagger}$ & 31 \\
\hline $\begin{array}{l}\text { False positive serological } \\
\text { test for syphilis }\end{array}$ & $1 / 24^{\dagger}$ & 4 & $19 / 129^{\dagger}$ & 15 \\
\hline Antinuclear antibody & $22 / 23^{+}$ & 96 & $174 / 175^{+}$ & 99 \\
\hline
\end{tabular}

${ }^{*} 0.05>p>0.01$ and ${ }^{* *} \mathrm{p}<0.01$ (standard error of difference between percentages ${ }^{10}$ ).

Indicates number of patients in whom the test was performed where this is less than the number in the study.

JOINTS AND MUSCLE

A peripheral non-deforming arthritis was the commonest clinical manifestation (81\%). Monarticular septic arthritis developed in two patients, and marked cachexia was a feature in seven patients $(23 \%)$.

\section{RENAL FEATURES}

Renal biopsies were performed in five patients; three of these showed changes typical of SLE, one was normal, and in one case an inadequate biopsy specimen was obtained. Raised blood urea or creatinine levels occurred in six patients, four of whom subsequently died.

\section{CARDIAC AND RESPIRATORY FEATURES} In addition to pleural and pericardial involvement there was one case each of myocarditis, endocarditis, cardiomyopathy, and rhythm abnormality (wandering pacemaker). A non-specific basal infil- tration on chest radiography associated with $a^{\frac{9}{4}}$ restrictive pattern on spirometry was found in two patients.

\section{NERVOUS SYSTEM}

Lumbar punctures were performed in five patients扂 with neurological manifestations; four were normal and one showed uniformly blood stained cerebro $-\infty$ spinal fluid with a xanthochromic supernatant com- $\overrightarrow{0}$ patible with a subarachnoid haemorrhage. Mononeuritis multiplex occurred in one patient.

MISCELLANEOUS FEATURES

Splenomegaly occurred in four patients $(13 \%)$ ander lymphadenopathy in 13 patients $(42 \%)$. Lympho node biopsies were performed in three patients; twos had non-specific changes and one showed a follicu- -9 lar malignant lymphoma (centrocytic/centroblastic).. This patient refused radiotherapy, defaulted treat $-\rightarrow$ ment with prednisolone and chlorambucil and, when seen a year later with septic skin lesions, had noclinical evidence of the lymphoma.

Two patients were treated for tuberculosis four $\overrightarrow{0}$ years and one year respectively before diagnosis of SLE, another patient received empirical antituberculous therapy for a pleural effusion with nos bacteriological or histological evidence of the dis $\frac{\partial}{2}$ ease, and two seriously ill patients were given a triap of antituberculous therapy before the diagnosis of SLE became apparent.

A polyclonal gammopathy was seen in seven of the eight patients who had immunoglobulin electrophoresis performed and, of the 14 patients whof had serum complement levels measured, nine hao. low C3 levels and seven low C4 levels.

COURSE AND MANAGEMENT

All patients received corticosteroids at some stage of the disease, and one patient required cyclophospha-3 mide and then azathioprine. Antibiotics and hyo potensive agents were used as appropriate. $\mathrm{Com}_{\supset}$ plications of prednisolone therapy included three cases of steroid induced diabetes mellitus and one patient who developed osteoporosis and collapse of a thoracic vertebral body.

There were nine deaths $(29 \%)$ during the study period. All the deaths occurred within one year of diagnosis, and five occurred within six weeks, giving a one year mortality rate of $35 \%$ (corrected for the 10 survivors followed up for less than one year). The? mean follow up of surviving patients was $15 \cdot 30$ months (range 1-71 months). Renal failure was. present in four of these patients, associated with septicaemia, diabetic ketoacidosis, peritonitis, and pericarditis respectively. Portal and superior mesen teric vein thrombosis caused the death of one 
patient, and another, who died within hours of admission to hospital, was found to have adhesive pericarditis and glomerulonephritis at autopsy. Postmortem examination of the patient with a wandering pacemaker who died suddenly showed pulmonary oedema, and an arrhythmia was postulated as the cause of death. The remaining two patients who died had cerebral involvement. Permission fc. postmortem examination was refused.

\section{Discussion}

In reviewing the literature, Christian found 10 year survival figures in SLE ranging from $50 \%$ to $90 \%$ and suggested $75 \%$ as an average. ${ }^{11} \mathrm{He}$ concluded that variations in reported survival figures are most strongly influenced by disease definition and clinical heterogeneity, with more chronic and milder disease patterns now being recognised. Patients with renal and neurological involvement have a poor prognosis, whereas those with prominent haematological manifestations fare relatively well. The high frequency of renal involvement in our patients $(71 \%)$ might, in part, explain the high mortality rate. Ballou et al investigated racial differences in 138 American patients with SLE and found that both chronic renal failure and death occurred more frequently in black than white patients. ${ }^{12}$ Kanyerezi et al thought their Ugandan patients might have a more severe form of the disease (renal involvement in $67 \%$ and mortality $19 \%$ ) and not that the milder cases were being misdiagnosed. ${ }^{7}$ The latter possibility does exist in countries such as Zimbabwe where much of the health care is delivered by ancillary health workers. Seedat and Pudifin attributed the high incidence of renal involvement in their series $(73 \%)$ to the late presentation of their patients. ${ }^{6}$ As the average time from onset of the first symptom to the diagnosis of SLE in all our patients was 10.6 months, but only 5.1 months in those with renal involvement, this seems unlikely to apply to our series.

Photosensitivity $(16 \%)$ and serositis $(23 \%)$ were relatively infrequent in our patients. Previous studies in black Africans ${ }^{5-7}$ have not recorded the incidence of photosensitivity, and it is possible that melanin has a protective role. Lymphopenia (less than $1500 / \mathrm{mm}^{3}\left(1.5 \times 10^{9} / \mathrm{l}\right)$ on two or more occasions) is a lesser known manifestation of SLE but was the most common haematological abnormality $(48 \%)$ in our patients. Since two patients were treated for tuberculosis before developing SLE this raises the possibility of drug induced lupus erythematosus, but the marked renal involvement in both cases makes this unlikely. Although the development of tuberculosis in any patient was not proved, one patient known to have SLE and two others, at the time undiagnosed, received antituberculous therapy because of their critical condition and the clinical possibility of tuberculosis. Feng and Tan report a tuberculosis prevalence rate of $5 \%$ in their 311 SLE patients in Singapore, ${ }^{13}$ and three of the 30 patients reported by Seedat and Pudifin developed tuberculosis while receiving immunosuppressive therapy. ${ }^{6}$ Feng and Tan emphasise the difficulty in diagnosing particularly extrapulmonary tuberculosis in patients with SLE, ${ }^{13}$ and diagnostic confusion between these two diseases will certainly continue in areas where tuberculosis is prevalent, marked cachexia being a common presentation of both. Septic arthritis occurred in one patient receiving prednisolone and another not receiving treatment and illustrates the importance of a high level of suspicion for this condition in patients known to have SLE.

In 1978 Green et al found 14 cases in the literature of lymphoma associated with SLE and reported four of their own. ${ }^{14}$ In 12 of these cases, as in our patient, SLE preceded the lymphoma. The lymphoma was of the non-Hodgkin variety in seven of these 12 cases. Our patient, and nine of the 12 reported by Green $e t$ $a l,{ }^{14}$ received no immunosuppressive therapy apart from corticosteroids, which are widely used in other conditions and not related to the development of lymphoid tumours. Suppressor $\mathrm{T}$ cell dysfunction allowing abnormal $\mathrm{B}$ cell proliferation in response to extrinsic or autoantigens has been suggested as a common factor underlying the association, with viral infections possibly playing a role in initiating the lymphoma. ${ }^{14}$ Despite the frequent occurrence of benign lymphadenopathy in SLE patients $(39 \%$ in our series), previous authors have stressed the importance of early lymph node biopsy. ${ }^{15}$ Spontaneous regression of low grade non-Hodgkin lymphomas is quite common. ${ }^{16}$ Venous and arterial thromboses are common in SLE patients with circulating lupus anticoagulant, ${ }^{17}$ so named for its ability to prolong phospholipid dependent coagulation tests with no correction on adding normal plasma, rather than from any propensity to cause excessive bleeding. ${ }^{18}$ Isolation of lupus anticoagulant is difficult but its presence is highly correlated with raised anticardiolipin levels, and the latter may be an indicator of SLE patients at high risk for thrombosis. ${ }^{18}$ Both our patients with thromboses were VDRL negative, but this test may not be sufficiently sensitive.

Bulawayo and Harare, with its satellite town of Chitungwiza, had a combined population of 1.24 million in $1982 .{ }^{19}$ Thus the ascertainment of 22 cases from these towns during the six year period 1979-84 gives an estimated incidence of three per million per 
year. This figure might be an underestimate to the extent that it is based on attenders at the study hospitals. Conversely, the incidence could be inflated by patients moving into the towns as a consequence of SLE development.

Although still an uncommon disease, SLE is being recognised more frequently in black Africans, and this paper illustrates the wide range of manifestations and relative severity of the disease in Zimbabwe. In 198412 of our 31 patients $(39 \%)$ presented for the first time. This may reflect a greater clinical awareness or changing patterns of disease.

\section{References}

1 Trowell H C. Non-infective disease in Africa. London: Arnold. 1960: 145.

2 Shaper A G. Systemic lupus erythematosus. East Afr Med J 1961: 38: 134-44.

3 Olweny C L. Systemic lupus erythematosus in a male Kenyan African. Makerere Med J 1961: XX: 15-8.

4 Greenwood B M. Autoimmune disease and parasitic infections in Nigerians. Lancet 1968: ii: 380-2.

5 Jessop S. Meyers O L. Systemic lupus erythematosus in Cape Town. S Afr Med J 1973; 47: 222-5.

6 Seedat Y K. Pudifin D J. Systemic lupus erythematosus in black and Indian patients in Natal. S Afr Med J 1977: 51: 335-7.
7 Kanyerezi B R. Lutalo S K. Kigonya E. Systemic lupus erythematosus: clinical presentation among Ugandan Africans. East Afr Med J 1980; 57: 274-8.

8 Gelfand M. 'Medical arthritis in African practice. Cent Afr Med J 1969: 15: 131-5.

9 Tan E M. Cohen A S. Fries J F, et al. The 1982 revised criteria for the classification of systemic lupus erythematosus. Arthritis Rheum 1982: 25: 1271-7.

10) Swinscow T D V. Statistics at square one. London: BMA. 1978: 28-9.

11 Christian C L. Systemic lupus erythematosus: clinical manifestations and prognosis. Arthritis Rheum 1982: 25: 877-8.

12 Ballou S P. Khan M A. Kushner I. Clinical features of systemic lupus ervthematosus-differences related to race and age of onset. Arthritis Rheum 1982: 25: 55-60).

13 Feng $\mathrm{P} \mathrm{H}$. Tan $\mathrm{T} \mathrm{H}$. Tuberculosis in patients with systemic lupus erythematosus. Ann Rheum Dis 1982; 41: 11-4.

14 Green J A. Dawson A A. Walker W. Systemic lupus erythematosus and lymphoma. Lancet 1978: ii: 753-6.

15 Milligan D W. Chang J G. Systemic lupus erythematosus and lymphoma. Acta Haematol (Basel) 1980; 64: 109-10.

16 McElwain T J. Solid tumours of the lymphoid system. Med Int 1983: 2: 1218-24.

17 Much J R. Herbst K D. Rapaport S I. Thrombosis in patients with the lupus anticoagulant. Ann Intern Med 1980; 92: 156-9.

18 Anonymous. Lupus anticoagulant [Editorial]. Lancet 1984: 1157-8.

19 Central Statistical Office. 1982 Population census: a preliminary assessment. Harare: Government Printers. 1984: 29. 